\title{
Correspondence
}

\section{Re: Musings on malaria morbidity and mortality after the new Mosquirix ${ }^{\circledR}$ vaccine}

Ghana Med J 2019; 53(3): 252-253 doi: http://dx.doi.org/10.4314/gmj.v53i3.10

We have read with interest the correspondence by Sackey HA in the Ghana Medical Journal. ${ }^{1}$ Dr. Sackey questions the relevance of ongoing pilot implementation and Phase IV studies of Mosquirix ${ }^{\circledR}$ vaccine in Ghana.

Importance of pilot implementation and Phase IV studies and pending questions: The Malaria Vaccine Implementation Project (MVIP) is coordinated by the World Health Organisation (WHO) and led by African health authorities in Ghana, Kenya and Malawi. In Ghana, the MVIP is led by Ministry of Health/Ghana Health Service and evaluated by a consortium of researchers from University of Ghana, University of Health and Allied Sciences, Agogo Malaria Centre, and the Research and Development Division of Ghana Health Service. The project is designed to address several outstanding questions related to the public health use of the vaccine. Additionally, Phase IV studies are ongoing to further assess the safety of the vaccine in Ghana (Kintampo and Navrongo) as a standard regulatory requirement for new vaccines. Indeed, this approach has been used for the introduction of other vaccines in Ghana such as the human papilloma virus vaccine. ${ }^{2}$ Specifically, the MVIP will provide data to the Ministry of Health and partners on how best to deliver the required four doses of the vaccine in routine settings; assess the vaccine's full potential role in reducing childhood deaths; and establish the vaccine's safety profile in the context of routine use. Since the Phase III study was not intended to measure the impact of the vaccine on mortality, the data from MVIP will confirm or refute the impact of the malaria vaccine on mortality as determined in the mathematical models outlined by Penny $\mathrm{M}$ et. al. ${ }^{3}$

In the Phase III trial, there was a non-statistical difference in the number of meningitis cases among children who received the malaria vaccine compared to those who did not receive the vaccine, $(11 / 2976,0.4 \%, 95 \%$ CI $0-0.7$ and $1 / 2974,0.0 \%, 95 \%$ CI $0-0.2$ respectively). 4 The cases of meningitis were not temporally related to vaccination and therefore was not considered by the regulatory authorities to be a barrier to real life implementation of Mosquirix ${ }^{\circledR}$. This observation is however, being further assessed in both the Phase IV study and MVIP.

Several questions, including those listed by Dr. Sackey's correspondence, were considered carefully by Ghanas Food and Drugs Authority (GFDA), European Medicine Agency (EMA), and World Health Organisation (WHO) based current knowledge and the quality of the Phase III data.
They concluded that the vaccine has a favourable risk/benefit profile and therefore the pending questions should not delay its controlled implementation because of the substantial benefit that can be realized - reduction of clinical malaria by $39 \%$, severe malaria by $29 \%$ and severe malaria anaemia by $62 \%{ }^{4}$

Longer term imact of Mosquirix ${ }^{\circledR}$. The question about longer term impact of Mosquirix ${ }^{\circledR}$ has recently been addressed by Tinto et. al. ${ }^{5}$ The study followed children who previously participated in the Phase 3 trial (from Nanoro, Burkina Faso; Kombewa, Kenya; and Korogwe, Tanzania) for an additional three years (a total of seven years). Results show that the incidence of severe malaria decreased with increasing age of the children, regardless of whether or not the children received the vaccine, and that there was no evidence of a rebound of severe malaria following the four-dose schedule of the vaccine. Over the entire seven-year post-vaccination period, vaccine efficacy against severe malaria was 36.7 percent $[95$ percent CI 14.6, 53.1] in children who started their four-dose vaccination schedule at the age of 5 to 17 months. Clinical malaria was reduced by 23.7 percent $[95$ percent CI 15.9 , $30.7]$ in this age group over the same period. In addition, the study did not raise new safety concerns, and the benefit/risk balance, as previously established during clinical trials, remains unchanged. ${ }^{5}$

Strengthening the malaria programme and new interventions: While the author of the correspondence is rightfully asking to strengthen the malaria control programme to ensure that the impact of the available interventions is maximized, it is also true that the new vaccine has the potential to provide substantial additional benefit on top of the currently available interventions. Indeed, the results of the Phase III studies were obtained in the context of high malaria control intervention uptake such as high use of insecticide treated nets and prompt access to malaria management. This confirms the fact that new interventions such as vaccines are required to further reduce the burden of malaria as the existing malaria prevention measures are imperfect. Indeed, the efficacy of insecticide treated nets is about $50 \%{ }^{6}$ and has its limits. The Ghana Health Service/Ministry of Health continues to scale up other malaria control measures using a multipronged approach informed by scientific evidence. The vaccine is not to replace the other malaria interventions but an add on. 
The pending questions raised by Sackey HA are actually the reasons why the Phase IV studies and the Malaria Vaccine Implementation Project (MVIP) are needed.

This could therefore be an opportunity to explain how new medicines and vaccines are developed and licenced. While Phase I-III studies are critical to primarily provide safety and efficacy data, they do have limitations. Indeed, Phase III trials are conducted under controlled conditions and do not provide all the data and information required for decision-making on widespread, everyday use. There are always questions that can only be addressed in real life settings. This approach is aptly described by O'Brien et. al. ${ }^{7}$ In summary, O'Brien et. al. describes a step wise pilot implementation to assess feasibility of the vaccines introduction amidst other partially effective malaria interventions such as long lasting insecticide treated nets and to collect further safety data as done for other vaccines in high income countries. Therefore, post approval studies such as Phase IV studies and pilot implementation are conducted to further assess safety, public health impact on morbidity/mortality and real-life vaccine introduction challenges. Hence the current MVIP constitutes an important step in providing additional data following Phase III trials conducted in Africa by leading African scientists and their collaborators.

\section{CONCLUSION}

Malaria still remains a significant public health burden in Ghana and other sub-Saharan African countries despite the use of current malaria prevention tools. The malaria vaccine introduced in Ghana and two other African countries has undergone a careful risk-benefit analysis by GFDA, Ghana's Ministry of Health/Ghana Health Service. The vaccine's potential to reduce clinical malaria, severe malaria and anaemia in moderate to high transmission areas such as Ghana outweighs identified adverse events and the need to wait any further for answers to a myriad of questions. The current Phase IV and MVIP studies are in accordance with drug development practices to further identify potential safety signals, determine public health impact and implementation feasibility.

Kwaku Poku Asante. MBChB, MPH, PhD. Director, Kintampo Health Research Centre, Ghana Health Service, Ghana

Email: Kwakupoku.asante@kintampo-hrc.org

Keziah Malm, MBChB, MPH, PhD. Programme Manager, National Malaria Control Programme, Ghana Health Service, Ghana

Email:keziah.malm@ghsmail.org
Delise Mimi Darko, BPharm, MBA

Chief Executive Officer, Food and Drugs Authority, Ghana

Email: mimidarko66@yahoo.co.uk

Kwadwo Ansah Koram, MBChB; PhD; FGCPS

Professor and Former Director

Noguchi Memorial Institute for Medical Research, College of Health Sciences, University of Ghana

Email:KKoram@noguchi.ug.edu.gh

\section{Conflict of Interest}

Dr. Asante's institution received research funds to conduct part of Phase II/ III trials of Mosquirix ${ }^{\circledR}$. Dr. Malm is the Programme manager for Malaria Control in Ghana. Other authors declare no conflict of interest

\section{REFERENCES}

1. Sackey HA Ghana Med J 2019; 53(2): 187-188

2. Wigle J, Coast E, Watson-Jones D. Human Papillomavirus (HPV) vaccine implementation in low and middle-income countries (LMICs): health system experiences and prospects. Vaccine 2013; 31 (37), 3811-3817

3. Penny MA, Verity R, Bever CA, Sauboin C, Galactionova K, Flasche S, White MT, Wenger EA, Van de Velde N, Pemberton-Ross $\mathrm{P}$, Griffin JT, Smith TA, Eckhoff PA, Muhib F, Jit M, Ghani AC. Public health impact and cost-effectiveness of the RTS,S/AS01 malaria vaccine: a systematic comparison of predictions from four mathematical models. Lancet. 2016; 387 (10016): 367-375.

4. RTS,S Clinical Trials Partnership Efficacy and safety of RTS,S/AS01 malaria vaccine with or without a booster dose in infants and children in Africa: final results of a phase 3, individually randomised, controlled trial. Lancet. 2015; 386 (9988): 31-45

5. Tinto H, Otieno W, Gesase S, Sorgho H, Otieno L, Liheluka E, Valéa I, Sing'oei V, Malabeja A, Valia D, Wangwe A, Gvozdenovic E, Guerra Mendoza Y, Jongert E, Lievens M, Roman F, Schuerman L, Lusingu J. Long-term incidence of severe malaria following RTS,S/AS01 vaccination in children and infants in Africa: an open three-year extension of a phase III randomized controlled trial. Lancet Infectious Diseases. 2019; 19 (8): 821 - 832

6. Lengeler C. Insecticide-Treated Bed Nets and Curtains for Preventing Malaria. Cochrane Database Systematic Review. 2004; (2):CD000363.

7. O'Brien KL, Binka F, Marsh K, Abramson JS. Mind the gap: Jumping from vaccine licensure to routine use. Lancet. 2016; 387 (10031). 1887-1889. 